\title{
Modelling granular soil to predict pressures on integral bridge abutments
}

\author{
J. Banks, T. Knight \& J. Young \\ Mott MacDonald, Croydon, United Kingdom
}

A. Bloodworth
University of Southampton, Southampton, United Kingdom

\begin{abstract}
Presented here is a granular soil model created to investigate the soil pressures which develop behind integral bridge abutments. The problem is introduced along with a brief summary of the fundamental behaviour, the model produced, and the initial validation. The paper looks at the initial outcome of the final validation procedure where experimental behaviour of an abutment is compared to the modelled behaviour, and the influence of the Young's modulus profile applied is investigated. The results show that the model behaves as expected producing lateral stresses close to those measured experimentally. Comparisons show that the Young's modulus profile adopted influences the resultant lateral stresses sufficiently to warrant further investigation.
\end{abstract}

\section{INTRODUCTION}

Traditionally, engineers have used bearings and expansion joints to accommodate bridge expansion and contraction caused by daily and seasonal temperature fluctuations. Studies carried out in the late 1980s showed that bearings and expansion joints have problems associated with durability (Wallbank 1989). Since the mid twentieth century Integral Bridges with no expansion joints or bearings have been used. Deck expansion and contraction is accommodated by movement of the abutments into the retained fill which eliminates the problem of durability but the movement of the abutments has been thought to cause a build up of lateral stresses, particularly in the case of full height abutments. In the United Kingdom BA42/96 (Highways Agency 2000) was issued and gave guidance on the soil pressures that should be adopted in design. The validity of the work on which the code of practice was based is a subject of continued debate by both researchers and practicing engineers. For this reason Integral Bridges have been used much less widely in the United Kingdom than conventional bridges.

As part of a strategy by the University of Southampton to further investigate the soil pressures $\mathrm{Xu}(2005)$ carried out radial controlled triaxial tests on granular material under cyclic loading. The strain levels and stress path used represented that typically experienced by an element of retained material behind an integral bridge abutment. This was the first time that the fundamental behaviour had been investigated in this manner.

The further research discussed in this paper builds upon this by use of numerical modelling. The behaviour of granular material under such loading was not represented by any available constitutive model. For this reason a model was developed based on this behaviour. The basis of the model is discussed here as is the validation process. The first stage of the validation process was implementation in a commercially available spreadsheet package. This was then used to develop a model in the Finite Difference Method package FLAC. Once this was implemented the triaxial tests were modelled and the results compared to those found experimentally. The final piece of validation used published laboratory data of a centrifuge test. The test rig was modelled in FLAC with the granular model used to represent the retained soil. 


\section{THE FUNDAMENTAL BEHAVIOUR}

The basis of the developed numerical model was the work of Xu (2005). Xu carried out radial strain controlled cyclic triaxial tests implementing a stress path typical of that for an element of soil, represented by Leighton Buzzard sand, behind an integral bridge. The element considered was a typical mid-height element at $4 \mathrm{~m}$ depth and the vertical cell pressure was kept constant at $80 \mathrm{kPa}$ to model the overburden stress to which the element would be subjected. The applicable radial strain range was estimated using both Finite Element analysis and a geostructural mechanism (Bolton \& Powrie 1988) which ensured that the element was under typical loadings as found in service. The specimens were brought to an initial at rest state prior to cycling commencing. Various strain ranges and densities were considered to ensure that the results were applicable to a range of bridges with different soil conditions.

The results of $\mathrm{Xu}$ showed that the typical relationship between horizontal stress and radial strain was that of a hardening law. As the sample was compressed the horizontal stress increased, and conversely upon triaxial extension the horizontal stress returned to the active state. When this was repeated for the same radial strain range in the following cycle the maximum horizontal stress was found to increase, as shown in Figure 1. Other key findings by Xu include:

- The soil densifies until a critical value is reached, at which point it dilates

- The soil stiffens, and peak horizontal stress increases, with cycles regardless of whether densification or dilatation occurs

- Axial strain varies dependant on cycles

$\mathrm{Xu}$ (2005) also investigated the same situation but replaced the sand with spherical glass ballotini. Under the same loading condition no build up of horizontal stress was found to occur. This allowed the researchers to conclude that the stress build up was primarily due to readjustment of the soil fabric due to rolling/sliding effects of non-spherical material close to the active state (Clayton et al. 2006). This is a significant finding as it concerns the fundamental behaviour of the soil.

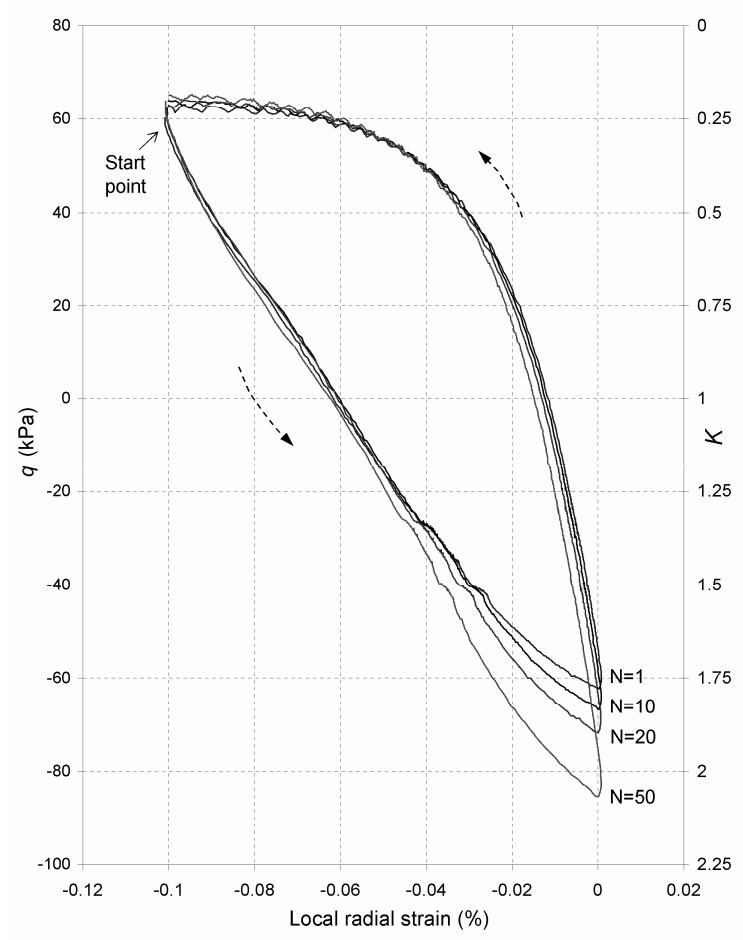

Figure 1. Typical Curve of Deviator Stress and Earth Pressure Coefficient K against Local Radial Strain for Leighton Buzzard Sand by Xu (2005). 


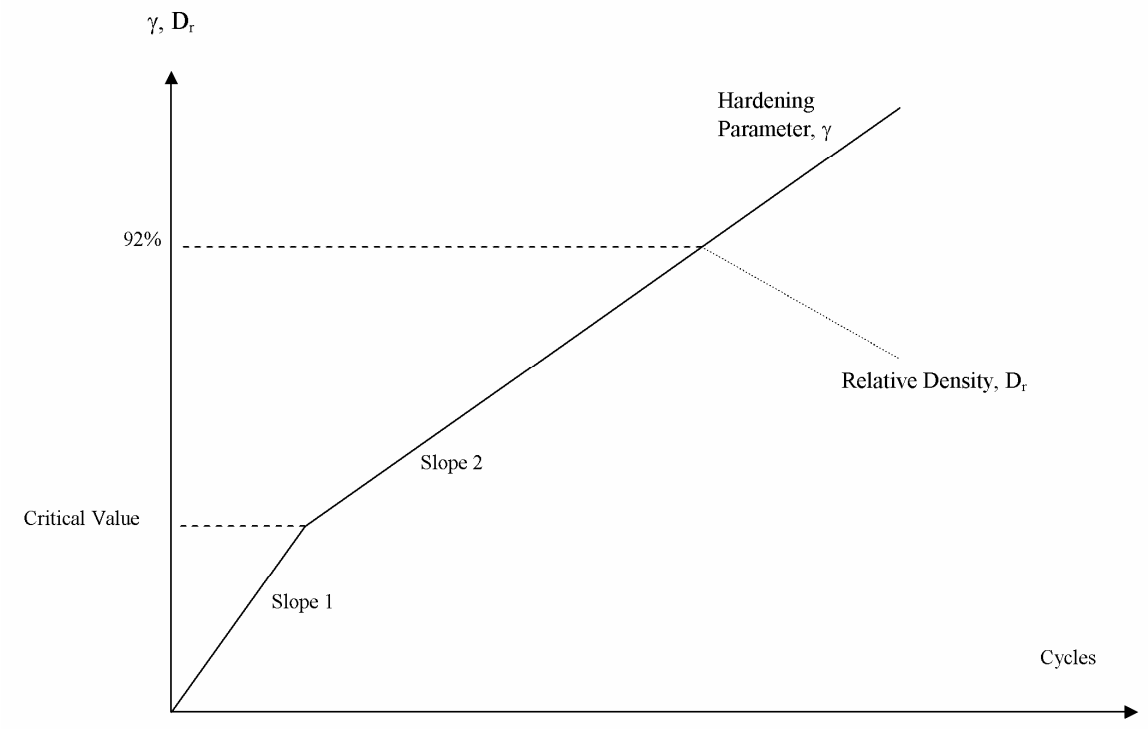

Figure 2. Development of the Hardening Parameter, $\gamma$, against cycles of Radial Strain.

\section{BASIS OF CONSTITUTIVE MODEL}

The fundamental behaviour described above was used to establish the model. It was noted that stiffness and axial strain was proportional to density until dilation occurred but then continued with a similar trend post-dilation. This indicated that the influence of rolling/sliding could not be underestimated. The work of Xu (2005) had quantified the densification of the soil but this was not possible with the effects of rolling/sliding and the two mechanisms could not be separated. To compensate for this the concept of a Hardening Parameter $(\gamma)$ was adopted. This was effectively a parameter which traced the density until the dilation point and then continued on the same slope beyond. This is shown in Figure 2.

As the hardening parameter relied on density it was essential to investigate the densification behaviour. It was found that the rate at which it densifies under cycles of constant strain has two distinct slopes. The point at which the change between slopes was noted was designated as the Critical value of Relative Density.

Therefore Equation 1 gives the change in relative density, $\Delta D_{r}$, as:

$$
\Delta D_{r}=f\left(\gamma, \Delta \varepsilon_{r}\right)
$$

where $\gamma=$ hardening parameter; and $\Delta \varepsilon_{r}=$ change in radial strain.

Similarly the critical value of relative density was found to be reliant on the radial strain range, giving:

$$
D_{\text {rcrit }}=f\left({ }_{\Delta \varepsilon_{r}}\right)
$$

where $D_{r c r i t}=$ critical relative density and $\Delta \varepsilon_{r}=$ change in radial strain.

$\mathrm{Xu}$ used Hooke's Law to derive the secant horizontal Young's modulus for each cycle. This was found to be represented by a logarithmic curve of the form given in Equation 3:

$$
E_{h}^{\prime}=A \ln \left({ }_{\Delta \varepsilon_{r}}\right)+B
$$

where $E^{\prime}{ }_{h}=$ Young's modulus; $\Delta \varepsilon_{r}=$ change in radial strain; and $A$ and $B=$ coefficients reliant on the hardening parameter and whether radial extension or compression is being considered.

Similarly the relationship between radial and axial strain was found to be of the form: 


$$
\Delta \varepsilon_{a}=X\left({ }_{\Delta \varepsilon_{r}}\right)^{3}+Y\left({ }_{\Delta \varepsilon_{r}}\right)^{2}+Z\left({ }_{\Delta \varepsilon_{r}}\right)
$$

where $\Delta \varepsilon \mathrm{a}=$ change in axial strain; $\Delta \varepsilon \mathrm{r}=$ change in radial strain; and $\mathrm{X}, \mathrm{Y}$ and $\mathrm{Z}$ are coefficients reliant on the hardening parameter and whether radial extension or compression is being considered. This relationship between radial and axial strain, although created based on data from $\mathrm{Xu}(2005)$ at a specific overburden stress $(80 \mathrm{kPa})$, was assumed to hold true at any value of overburden pressure.

These relationships could then be used in conjunction with Hooke's Law to calculate the stresses in the soil. However, before this could be tested the onset of failure needed to be considered. Values of the stress invariants $p$ ' and $q$ ' were plotted giving the stress path intended by $\mathrm{Xu}$. The failure criterion from Critical State Soil Mechanics was considered. The failure surface of gradient $M$, where $M$ is a function of the estimated angle of internal friction, was then superimposed on the plotted values. It was found from this that the soil failure coincided with the surface and therefore the decision was made to adopt this failure criterion.

\section{VALIDATION BY TRIAXIAL TEST}

The next stage was to implement the constitutive model described above into the commercial finite difference method (FDM) package FLAC (Itasca 2005). There is considerable expertise in FLAC within the Mott MacDonald group and FLAC has the built in programming pseudolanguage 'Fish' (FLAC-ish) making it possible to program user-defined constitutive models.

A model of a triaxial test was created using a single axi-symmetric element, and the constitutive model was assigned with the properties and initial loading conditions based on the experiments by $\mathrm{Xu}(2005)$, with an overburden of $80 \mathrm{kPa}$ to simulate a typical element at $4 \mathrm{~m}$ depth, and at rest horizontal stresses. The analysis was run for many cycles, and a typical example of the lateral stress output is shown in Figure 3, showing that the model worked as expected.

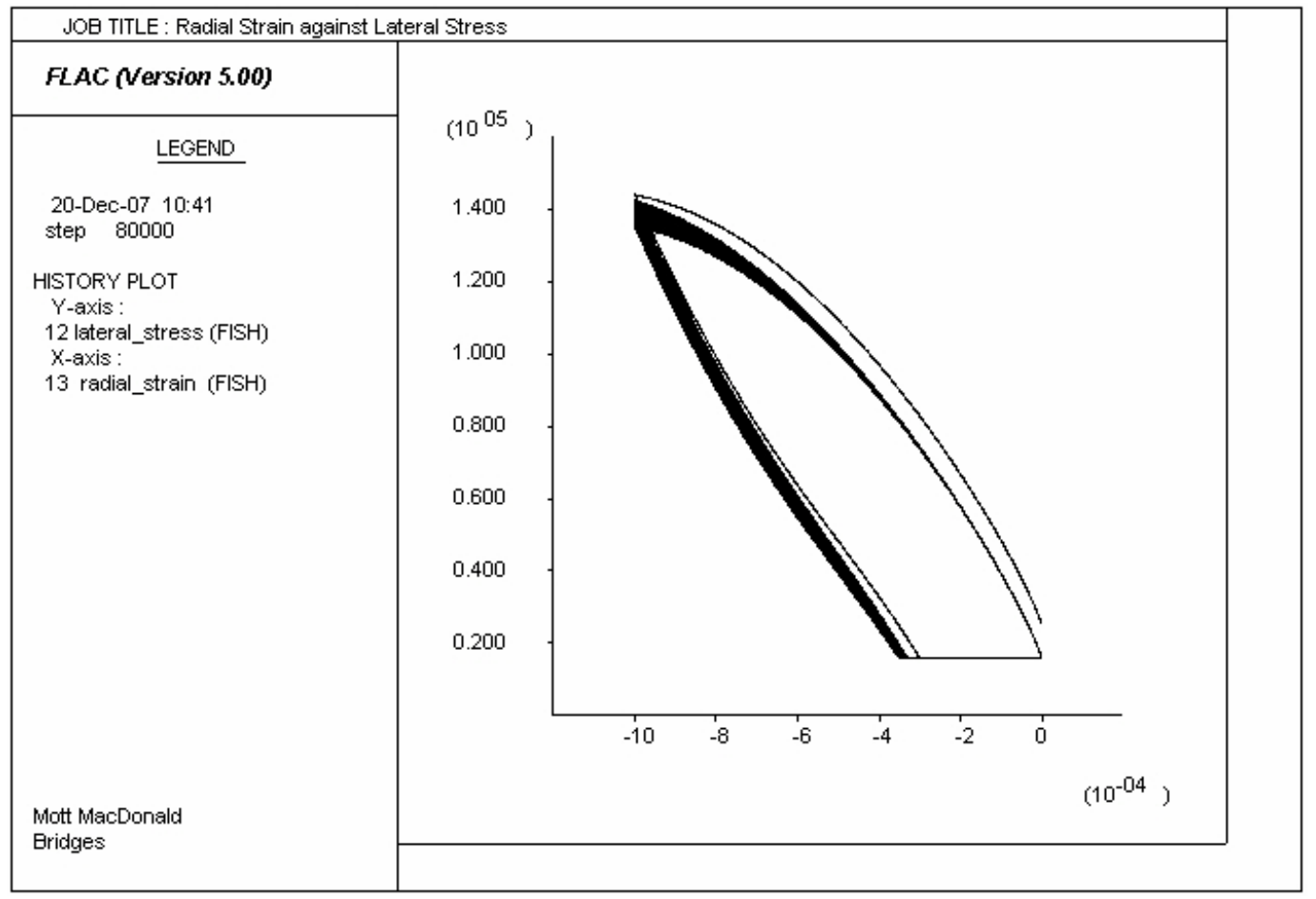

Figure 3. Typical Curve of Lateral Stress against Radial Strain from FLAC analysis of the triaxial test using the granular constitutive model. 


\section{VALIDATION USING EXPERIMENTAL DATA}

The triaxial test modelling showed that the mathematical model as implemented and programmed within FLAC worked for a single element. The next stage of validation was to investigate how the constitutive model behaved when applied to a larger system consisting of an abutment structure and retained soil. An initial series of analyses were carried out and are reported on in this paper in which the results were to be compared with experimental laboratory data for an abutment. Further research is under way to more fully explore the capabilities of the model and carry out parametric studies.

\subsection{Tapper and Lehane experiments}

Tapper \& Lehane (2004) carried out centrifuge testing using a 1:20 scale model of a stiff abutment wall with a pinned base. Medium dense uniform siliceous sand was used as the backfill. Three rotation amplitudes of the wall were investigated and the results show that a steady state in terms of pressure coefficient behind the wall was not reached even after 1000 cycles, which is similar to the findings of Xu (2005) in his triaxial tests. Therefore, the work of Tapper and Lehane was regarded as appropriate for validation purposes.

The dimensions of the prototype on which the centrifuge model was based were used in the FLAC validation model rather than the centrifuge test specimen itself. The prototype consisted of a stiff wall pinned at the base and propped at the top, with the prop moved by a distance $\Delta$ either side of a zero position to imitate the thermally induced bridge deck movements. The retained height $H$ of the granular material behind the wall was $4 \mathrm{~m}$, and the prototype was $10.6 \mathrm{~m}$ long, ensuring that the remote vertical boundary would not influence the stress development at the back of the abutment.

\subsection{Model}

A plane strain FLAC model was created with the same dimensions as the prototype. The bottom boundary was fixed against vertical movement, and the remote vertical boundary was fixed horizontally. The wall is described as "stiff" in the published data so the second moment of area and Young's modulus were set for infinite wall stiffness. The wall was modelled as a series of beam elements with a frictional interface to the soil, with the wall friction set to half the angle of internal friction of the soil as prescribed in BA42 (Highways Agency 2000). The wall was pinned at its base as in the prototype. The displacement $\Delta$ was applied at the top of the wall as a velocity over 50 timesteps.

The grid generated in FLAC was 8 elements high by 20 elements wide (Fig. 4), with all elements of the same size. Although it would be usual to reduce the element size in the area of interest, in this case at the back of the wall, this was not done in this case because:

- The model was not being used to generate design data but rather to look at trends only and to indicate if the model was behaving as expected.

- User defined models in FLAC run at about a quarter to a third of the speed of self contained models so keeping the elements to a minimum allowed a quicker run time.

An initial density of $53 \%$ was assigned to the material, and cycles of rotation of amplitude $\Delta / \mathrm{H}$ equal to $0.2 \%$ were applied to the model.

There were two aims of these initial tests. The primary aim was to investigate whether the model would run and whether it behaved in a manner expected, i.e. whether $\gamma$ and the lateral stress on the wall increased with cycles. With this in mind, Test A was run to 100 cycles and the maximum lateral stresses for the $5^{\text {th }}$ and $100^{\text {th }}$ cycle recorded and plotted against those found experimentally by Tapper and Lehane.

The tests also aimed to assess the sensitivity of the model to the variation of Young's modulus of the soil. The triaxial tests by $\mathrm{Xu}$ (2005) calculated the value of secant horizontal Young's modulus based on an overburden of $80 \mathrm{kPa}\left(E^{\prime}{ }^{\prime}{ }^{80000}\right)$. Because the maximum vertical stress calculated in the soil mass in Tapper and Lehane's prototype was $63 \mathrm{kPa}$, the Young's modulus had to be reduced accordingly by a factor of 0.8 . Since it is also common to vary Young's modulus linearly with depth, three tests of the model, B1, B2 and B3 (Fig. 5) were car- 
ried out to investigate the sensitivity of the model to different schemes for achieving this variation with depth. It was hoped that this would indicate if a larger parametric study would be necessary. For each test B1 $-\mathrm{B} 3$, the $1^{\text {st }}, 5^{\text {th }}, 50^{\text {th }}$ and $100^{\text {th }}$ cycle was considered and the results compared.

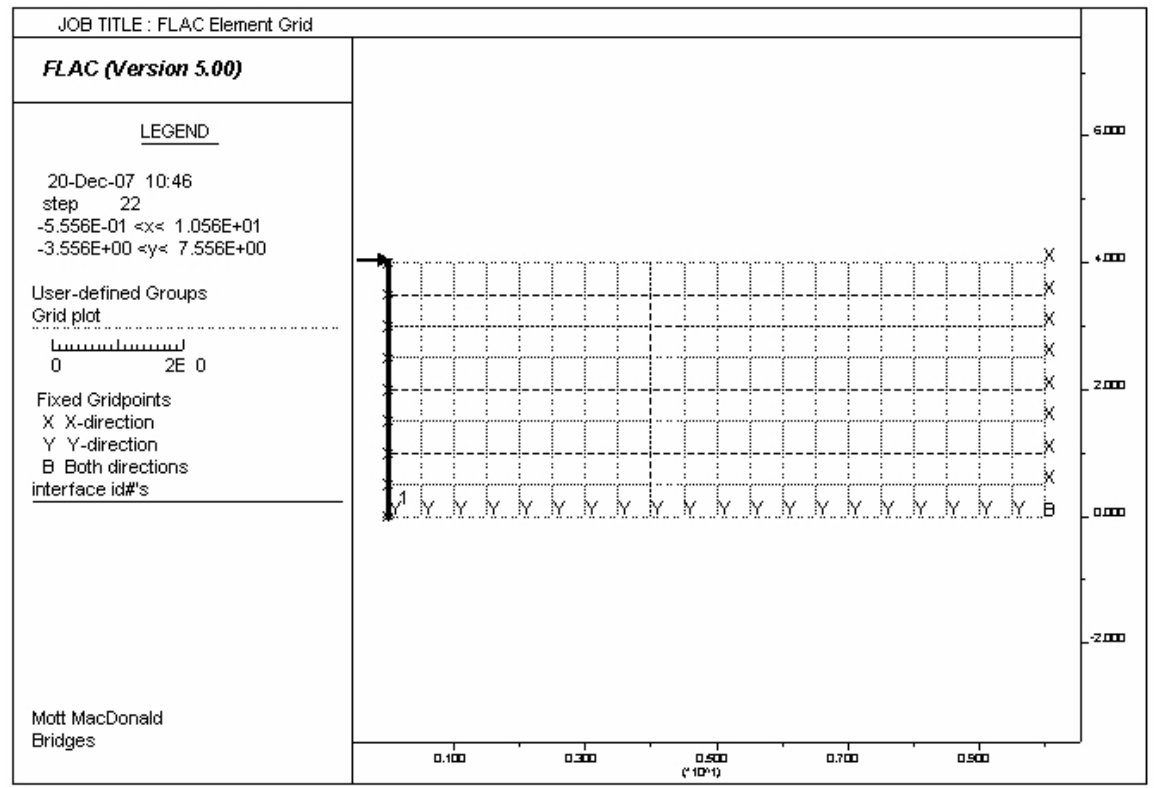

Figure 4. Grid used in FDM analysis.
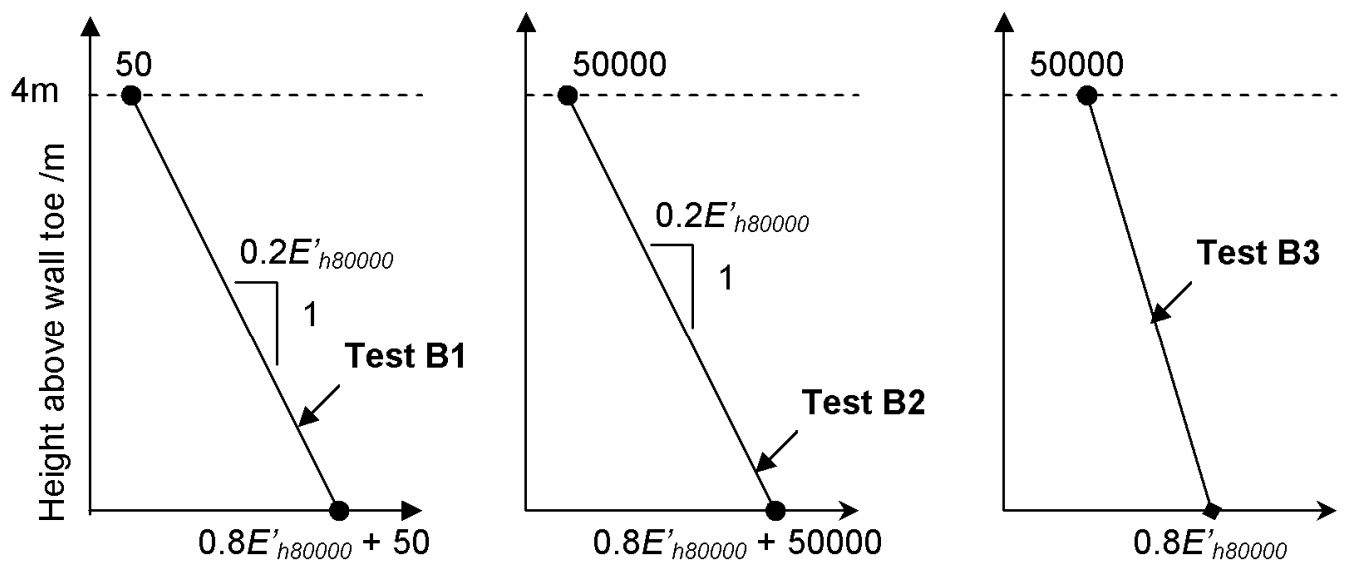

Secant horizontal Young's modulus $\mathrm{E}_{\mathrm{h}}^{\prime} / \mathrm{kN} / \mathrm{m}^{2}$

Figure 5. Profiles of Young's modulus with depth used in the test series B.

\subsection{Initial Results}

Figure 6 shows a plot of measured lateral stress against height above wall toe for both Test A and the work of Tapper \& Lehane (2004). Comparing the $1^{\text {st }}$ and $100^{\text {th }}$ cycles shows that firstly, the model is providing stresses in the expected range, although in the model, they have increased only slightly above at rest pressures when the $5^{\text {th }}$ cycle is reached and at that stage had not yet exhibited the "triangular" envelope which develops in the experimental case. 
By the time the $100^{\text {th }}$ cycle was reached, the stresses in the model are approaching those of Tapper and Lehane, although still underestimated. The stress envelope has become triangular in profile as in the experimental case. The general trends at each measuring point also indicate the model is behaving well at these positions. At $h=1 \mathrm{~m}$ there is only minimal change in lateral stress. Further up, close to the mid height position $(h=2.25 \mathrm{~m})$, the change in maximum lateral stress between the $5^{\text {th }}$ and $100^{\text {th }}$ cycles is at its greatest value as in the experimental case. In each case the increment in maximum lateral stress between the $5^{\text {th }}$ and $100^{\text {th }}$ cycles is similar in the experimental and model predicted case. Considered in a design context, the development of a triangular envelope causes the load to be placed relatively distant from the centre of rotation. Ultimately this causes high bending loads in the abutment which the structure must be designed to resist.

Figure 7 shows typical maximum lateral stress profiles after 100 cycles of the wall for the series of tests $\mathrm{B} 1-\mathrm{B} 3$.

Comparing Test B1 and Test B2, which have the same modulus profile gradient but different surface values, shows that variation in Young's modulus profile can make a significant difference to the results, especially for example at the surface where the vertical stress is low, due to the value of Young's modulus being directly proportional to the overburden stress.

Test B2 and Test B3 have the same surface values of Young's modulus but with different gradients. Near the surface the actual value of Young's Modulus is very similar and the resulting lateral stresses reflect this with comparable values. As depth increases the predicted lateral stress profiles diverge, with a maximum difference occurring near mid height of the soil mass. Below this point, the two lateral stresses converge. This comparison shows that a change in gradient of the applied Young's Modulus profile can also have an effect on the lateral stresses that develop.

The comparison of these three tests proves that careful consideration must be given to the profile of Young's modulus employed in any modelling. Variations can occur due both the surface value of Young's Modulus and gradient applied and in both cases this is particularly the case at low depths, meaning that this effect may have a significant impact on the bending stresses which develop within the abutment. The results described here allow the conclusion to be drawn that this requires further research, in the form of a parametric study, to allow future modelling to be sufficiently accurate.

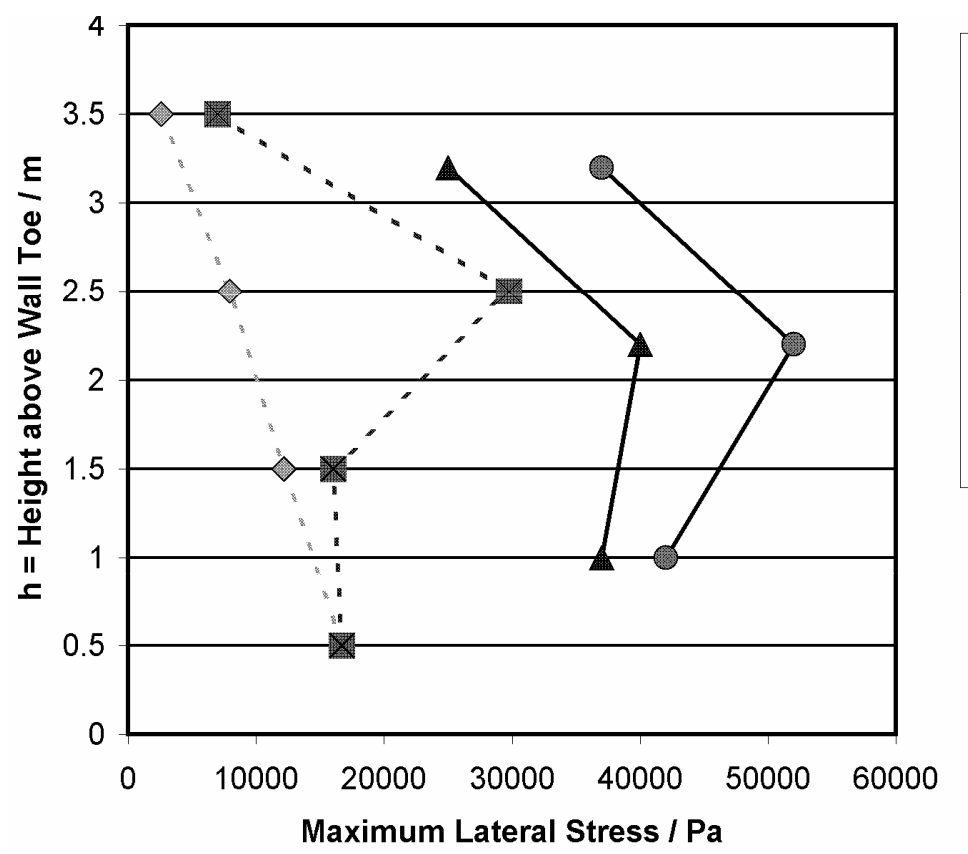

\begin{tabular}{|l}
\hline - Tapper and Lehane - \\
Cycle 5 \\
- Tapper and Lehane \\
Cycle 100 \\
Granular Model - \\
Cycle 5 \\
- Granular Model - \\
Cycle 100
\end{tabular}

Figure 6. Lateral Stress against Height Comparison of Granular Model Prediction against experimental data of Tapper \& Lehane (2004). 


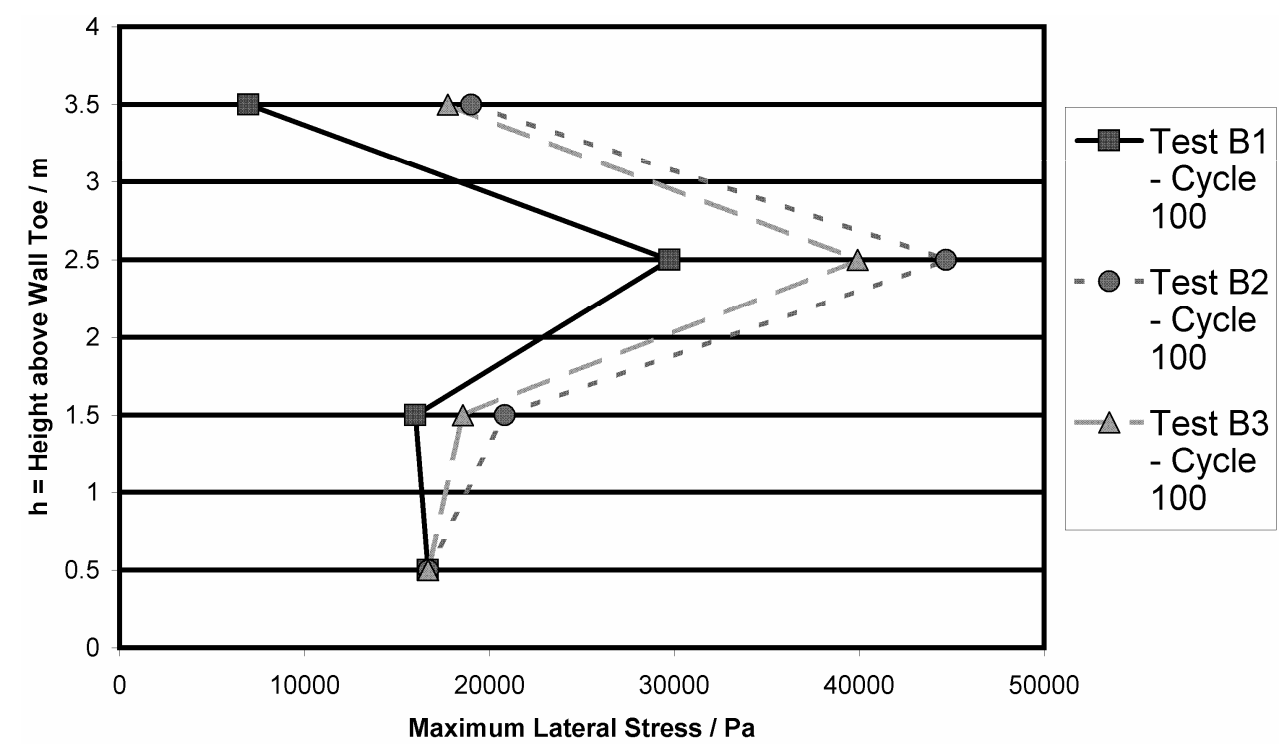

Figure 7. Lateral Stress after 100 cycles against Height Comparison for different Young's modulus Profiles.

\section{CONCLUSIONS}

The advantages of using Integral Bridge construction have been widely publicised, but equally the disadvantages associated with lateral soil stress on the abutments and their potential to build up cannot be neglected. The work of Xu (2005) showed the fundamental behaviour associated with soil behind an integral bridge, behaviour used in the subsequent modelling work reported in this paper to develop a granular soil constitutive model capable of predicting the effect of the cyclic loading the soil experiences in this situation.

This paper has described the implementation of this model into an FDM code and its use to model a centrifuge prototype abutment. It has been shown that the model behaves well up until the $100^{\text {th }}$ cycle, predicting a lateral stress profile and magnitude similar to the experimental observations. It has also been demonstrated that the Young's modulus profile adopted has a significant effect on the results achieved; indicating the importance of this parameter and the need for further investigation before modelling of bridges is carried out. However, the paper also proves that the type of constitutive model developed here has the potential to provide soil pressure estimates and could be used to further investigate integral bridge abutment loads.

\section{REFERENCES}

Bolton, M.D. \& Powrie, W. 1988. Behaviour of diaphragm walls in clay prior to collapse. Géotechnique, 38(2): 167-189.

Clayton, C.R.I, Xu, M. \& Bloodworth, A. 2006. A laboratory study of the development of earth pressure behind integral bridge abutments. Géotechnique, 56(8): 561-571.

Highways Agency. 2000. BA 42/96 The design of integral bridges - incorporating amendment 1, DMRB 1.3, London: HMSO

Itasca Corporation. 2005. FLAC Command and FISH Reference Summary, $3^{\text {rd }}$ Edition (FLAC Version 5.0) April 2005. Minneapolis: Itasca

Tapper, L. \& Lehane, B.M. 2004. Lateral stress development on integral bridge abutments. In A.J. Deeks and H. Hao (eds.), Proceedings of the Eighteenth Australasian Conference on Mechanics of Structures and Materials, Perth, 1-3 December 2004. Rotterdam: Balkema

Wallbank, J. 1989. The performance of concrete in bridges: a survey of 200 highway bridges, London: HMSO

Xu, M. 2005. The Behaviour of Soil behind Full-height Integral Abutments, PhD thesis, University of Southampton 